\title{
Zel'dovich Cosmological Model in Lyra Manifold
}

\author{
V.G. Mete \\ Department of Mathematics, R.D.I.K. \& K.D. College, Badnera -Amravati, India \\ Email: vmete5622@gmail.com
}

\begin{abstract}
This paper deals with the study of five dimensional non - static plane symmetric cosmological model within the framework of Lyra's manifold when the source of the gravitational field is perfect fluid with pressure equal to energy density. The cosmological solutions have been analyzed through various physical and geometrical properties.
\end{abstract}

Keywords: Plane symmetry, higher dimension, Lyra geometry.

\section{Introduction}

After the formulation of general relativity (GR) by Einstein, many alternative geometric theories have been developed in order to explain gravitation phenomena. Inspired by geometrizing gravitation, Weyl [1] proposed a more general theory in which both gravitation and electromagnetism are described geometrically. For a long time, Weyl's theory was not taken seriously due to non-integrability of length of vector under parallel displacement.

Lyra [2] proposed a new modification of Riemannian geometry by introducing a gauge function which removes the non-integrability condition of the length of a vector under parallel transport. This modified Riemannian geometry is known as Lyra's geometry.

In consecutive investigations, Sen [3] and Sen and Dunn [4] proposed a new scalar tensor theory of gravitation and constructed an analog of Einstein's field equations based on Lyra's geometry which in normal gauge may be written as

$$
R_{i j}-\frac{1}{2} g_{i j} R+\frac{3}{2} \varphi_{i} \varphi_{j}-\frac{3}{4} g_{i j} \varphi_{k} \varphi^{k}=-8 \pi T_{i j}
$$

where $\varphi_{i}$ is a displacement field and the other symbols have their usual meaning as in Riemannian geometry.

According to Halford [5], the present theory predicts the same effects within observational limits, as far as the classical solar system tests are concerned. Soleng [6] has pointed out that the constant displacement field in Lyra's geometry will either include a creation field and be equal to Hoyle's creation field cosmology or contain a special vacuum field which together with the gauge vector term may be considered as a cosmological term.

Several authors [7-16] have investigated various aspects of cosmological models in scalar tensor theory and cosmology within the framework of Lyra's geometry. In general relativity a non-static plane symmetric Zel'dovich universe has been obtained by Reddy and Innaiah [17]. Pradhan et.al [18] studied LRS Bianchi-I cosmological models, Singh [19] obtained a new exact solutions for local cosmic string in Lyra manifold.

The unification of gravitational forces with other forces in nature is not possible in the usual four dimensional space times. Hence higher dimensional theory might be useful at early stages of the evolution of the universe. In fact, as time evolves, the standard dimensions expand while the extra dimensions shrink to the planckian dimension, which is beyond our ability to detect with the currently available experimental facilities Chatterjee et. al [20]. This fact has attracted many researchers to investigate the problems in the field of higher dimensions. Appelquist et .al [21], Rahaman et.al [22] studied higher dimensional spherically symmetric perfect fluid model in Lyra geometry, Khadekar et.al [23] constructed five dimensional FRW cosmological model with quark and strange quark matter. Five dimensional Bianchi type-I string cosmological model in Lyra manifold have been investigated by 
Samanta et.al [24].Recently Katore et. al [25] constructed Kaluza Klein universe with magnetized anisotropic dark energy in the context of Lyra manifold.

Motivated by the above discussions, in this paper, we have constructed a five dimensional non-static plane symmetric Zel'dovich cosmological model based on Lyra's geometry.

\section{Plane Symmetric Zel'dovich Universe}

Here we consider the Riemannian space-time described by the line element (Bera 1969) in five dimensions.

$$
d s^{2}=e^{2 h}\left[d t^{2}-d r^{2}-r^{2} d \theta^{2}-S^{2}\left(d z^{2}+d w^{2}\right)\right]
$$

where $r, \theta, z$ and $w$ are the usual cylindrical polar coordinates and $h$ and $s$ are functions of $t$ only. It is well known that this line element is a plane symmetric.

Zel'dovich fliud can be regarded as a perfect fluid having the energy - momentum tensor

$$
T_{j}^{i}=(\rho+p) V^{i} V_{j}-\delta_{j}^{i} p
$$

characterized by equation of state

$$
\rho=p
$$

where $\rho$ is the energy density and $p$, the isotropic pressure of the matter and $v^{i}$ is the four velocity vector of the fluid, we use comoving coordinates so that

$$
V^{1}=V^{2}=V^{3}=V^{4}=0 \text { and } V^{5} V_{5}=1
$$

We assume the vector displacement field $\varphi_{i}$ in equation $(1)$ to be the time like constant vector

$$
\varphi_{i}=(0,0,0,0, \beta)
$$

where $\beta$ is a constant.

For the metric (2), the field equations (1), with the help of equations (3) to (6) can be written, explicitly, as

$$
\begin{gathered}
e^{-2 h}\left[3 h_{55}+3 h_{5}^{2}+\frac{6 h_{5} s_{5}}{s}+\frac{2 s_{55}}{s}+\frac{s_{5}^{2}}{s^{2}}+\frac{3}{4} \beta^{2}\right]=-8 \pi p \\
e^{-2 h}\left[3 h_{55}+3 h_{5}^{2}+\frac{3 h_{5} s_{5}}{s}+\frac{s_{55}}{s}+\frac{3}{4} \beta^{2}\right]=-8 \pi p \\
e^{-2 h}\left[6 h_{5}^{2}+\frac{6 h_{5} s_{5}}{s}+\frac{s_{5}^{2}}{s^{2}}-\frac{3}{4} \beta^{2}\right]=8 \pi p
\end{gathered}
$$

The suffix 5 after $h$ and $s$ denotes ordinary differentiation with respect to $t$.

The equations (7) to (9) represent the field equations for Zel'dovich fluid distribution in Lyra manifold. After a proper choice of coordinates, field equations (7-8) yield the cosmological model given by

$$
d s^{2}=T^{(1-a)}\left[d T^{2}-d R^{2}-R^{2} d \theta^{2}-T^{2 a}\left(d z^{2}+d w^{2}\right)\right]
$$

where $a$ is a positive constant satisfying

$$
(3+a)(1-a)=32 \pi C_{o}, \quad a<1
$$

The model (10) is quite similar to the model obtained by Reddy and Innaiah [26] in general relativity and Reddy et.al [13] in Saez-Ballester (1985) scalar-tensor theory of gravitation.

\section{$3 \quad$ Some Physical Properties of the Model}

The pressure $p$ and density $\rho$ in the model (10) are given by

$$
p=\rho=\frac{1}{32 \pi}\left[(1-a)(3+a)-3 \beta^{2} T^{2}\right] T^{a-3}
$$

on physical grounds $\rho>0, p>0$, we get 


$$
0<a<1, T>0
$$

and

$$
(1-a)(3+a)>3 \beta^{2} T^{2} .
$$

It may be observed that if $a=-3$ or $a=1$ and $\beta=0$, the model reduces to empty space-time discussed by Bera (1969).

The flow vector $V^{i}$ of the distribution is given by

$$
V_{1}=V_{2}=V_{3}=V_{4}=V^{1}=V^{2}=V^{3}=V^{4}=0
$$

and

satisfies the equations of geodesics

$$
V_{5}=T^{\frac{1-a}{2}}, V^{5}=T^{\frac{a-1}{2}}
$$

$$
V_{; j}^{i} V^{j}=0
$$

Thus the lines of flow in the model are geodesics.

The Physical and Kinematical parameters for the model (10) are Spatial Volume:

$$
V_{;}^{4}=(-g)^{\frac{1}{2}}=r \cdot T^{\frac{5-a}{2}}
$$

Expansion Scalar:

$$
\theta=\frac{1}{4} V_{; i}^{i}=\frac{1}{2} T^{\frac{a-3}{2}}
$$

Shear Scalar:

$$
\sigma^{2}=\frac{1}{2} \sigma_{i j} \sigma^{i j}=\frac{1}{8}\left(a^{2}-2 a+5\right) T^{a-3}
$$

where

$$
\sigma_{i j}=\frac{1}{2}\left(V_{i ; j}+V_{j ; i}\right)-\theta\left(g_{i j}-V_{i} V_{j}\right)
$$

It is observed that for $a=0$, the model becomes conformal to flat space-time and reduces to a particular case of Lemaitre universe. It can be seen that there is no initial singularity in the model. Also pressure and energy density are not singular at $T=0$. For $a=3$, the expansion scalar $\theta$ vanishes while the energy density and shear scalar become constant.

Also for $a \neq 3$

$$
\lim _{T \rightarrow \infty}\left(\frac{\sigma}{\theta}\right)=\frac{1}{\sqrt{2}}\left(a^{2}-2 a+5\right)^{\frac{1}{2}}
$$

the model is not isotropic for large $T$.

The deceleration parameter $q$ is given by Fienstein at al. [27]

$$
q=-3 \theta^{2}\left[\theta ; \alpha V^{\alpha}+\frac{1}{3 \theta^{-2}}\right]
$$

For the model (10), we have $q=-10$ (when $a=1$ ) and $q=2$ (when $a=-3$ ) which indicates that the model accelerates and decelerates in standard way.

\section{Conclusion}

In this paper, we have studied non-static plane symmetric Zel'dovich cosmological model in Lyra's manifold in higher dimensions.

Non-static universe plays an important role in understanding of cosmological and astrophysical significance. The plane symmetric cosmological models in general relativity are of considerable importance [28-30]. Here we have considered a non-static plane symmetric metric given by Bera (1969) and obtained a cosmological model in Lyra manifold, which represents a Zel'dovich universe in higher 
dimensions. It is observed that the universe is free from initial singularity. It is interesting to note that, our investigation in absence of fifth dimension resembles to that of Reddy et. al [26].

Acknowledgement. The author would like to acknowledge his deep sense of gratitude to the anonymous referee whose valuable comments and suggestions have improved this research manuscript.

\section{References}

1. H. Weyl and S ber, Preuss. Akad. Wiss. (Berlin) 465, 1918.

2. G. Lyra, Math. Z. 54, 52, 1951.

3. D.K. Sen, Z. Phys. 149, 311, 1957.

4. D.K. Sen and K.A.Dunn, J. Math. Phys. 12, 578,1971 .

5. W.D, Halford, Aust. J. Phys. 23, 863,1970

6. H.H Soleng, Gen. Relativ. Gravit. 19, 1213,1987.

7. K.S,Bhamra, Aust. J. Phys. 27, 541,1974 .

8. S.B.Kalyanshetti and B.B. Waghmode, Gen. Rel. Grav. 14, 823, 1982.

9. D.R.K. Reddy and P. Innaiah, Astrophys. Space Sci. 114, 285, 1985.

10. D.R.K Reddy and R.Venkateswarlu, Astrophys. Space Sci. 136, 183, 1987.

11. A. Beesham, Astrophys. Space Sci. 127, 355, 1986.

12. A. Beesham, Aust. J. Phys. 41, 833-842, 1988.

13. D.R.K. Reddy, R. Venkateswarluand and Ch. C.S.V.V.R. Murthy., Journal of the Indian Mathematical Society, 68, 145, 2001.

14. D.R.K.Reddy and R.Vekateswarlu, Astrophysics and Space Science, 149, 287, 1988.

15. A.Pradhan, H. Amirhashchi and H. Zainuddin, Int.J.Theor.Phys.50, 1,56-69,2011.

16. S. Agarwal, R.K.Pandey and A.Pradhan, Int.J.Theor.Phys.50, 1,296-307, 2011.

17. D.R.K Reddy and P.Innaiah, Einstein Foundation International, 2,9 ,1985.

18. A.Pradhan, A.K. Vishwarkarma, Journal of Geometry and Physics, 49, 3-4,pp.332-342,2004.

19. J.K. Singh, Astrophys. Space Sci. 317, 39-44, 2008.

20. S.Chatterji, B. Bhui, and A. Banerjee, Physics Letters A , 149, No.2-3,91-94 ,1990.

21. T.Appelquist, A. Chodas and P.G.O Freund, "Modern Kaluza Klein theories" (Addison Wesley, Bostan, 1987).

22. F.Rahaman, S. Das, N. Begum, and M. Hossain, Pramana J.Phys.,61,1,153-159 2003.

23. G.S.Khadekar and R. Shelote, Int.J.Theor.Phys., 51(5), 1442-1447, 2011.

24. G.C.Samanta, S.Debata, Journal of Modern Physics, 3, 180-183, 2012.

25. S.D.Katore, S.P.Hatkar,New Astronomy, 34, 172-177,2015.

26. D.R.K .Reddy,R.L. Naidu, J. Indian Maths. Soc., 72, Nos.1- 4,47-51,2005.

27. A.Feinstein, J. Ibanez and P. Labarge, J.Math.Phys.36, 4962,1995.

28. A.H. Taub, Annals of Mathematics, $33,472,1951$.

29. K.P. Singh and D.N. Singh, Monthly Notices of Royal Astronomical Society, $140,453,1968$.

30. S.R. Roy and O.P. Tiwari, Indian Journal of Pure and Applied Mathematics, 14(2), 233 ,1983. 